\title{
A corrected and generalized successive random additions algorithm for simulating fractional Levy motions ${ }^{1}$
}

\author{
Hui-Hai Liu², Gudmundur S. Bodvarsson ${ }^{2}$, Silong $\mathrm{Lu}^{3}$ and Fred J. Molz
}

To be submitted to Mathematical Geology for publication

(Suggested running head: Corrected SRA for fLm)

\footnotetext{
${ }^{1}$ Received ; Accepted

${ }^{2}$ Earth Sciences Division, Lawrence Berkeley National Laboratory, Berkeley, CA 94720; email: hhliu@lbl.gov.

${ }_{3}$ Environmental Engineering and Science Department, Clemson University, Clemson, SC 29634.
} 
Simulation of subsurface heterogeneity is important for modeling subsurface flow and transport processes. Previous studies have indicated that subsurface property variations can often be characterized by fractional Brownian motion (fBm) or (truncated) fractional Levy motion (fLm). Because Levy-stable distributions have many novel and often unfamiliar properties, studies on generating fLm distributions are rare in the literature. In this study, we generalize a relatively simple and computationally efficient successive random additions (SRA) algorithm, originally developed for generating Gaussian fractals, to simulate fLm distributions. We also propose an additional important step in response to continued observations that the traditional SRA algorithm often generates fractal distributions having poor scaling and correlation properties. Finally, the generalized and modified SRA algorithm is validated through numerical tests.

KEY WORDS: Levy Fractal, Heterogeneity, Numerical simulation. 


\section{INTRODUCTION}

It is generally agreed that heterogeneity of hydraulic properties is a key factor controlling flow and transport processes in the subsurface. Consequently, quantification of subsurface heterogeneity is often needed for modeling subsurface contamination and remediation problems. Previous research has shown that spatial variations of K (hydraulic conductivity), or $\log (\mathrm{K})$ often display scaling that has been modeled using properties of truncated fractional Levy motion (fLm) (Painter and Paterson, 1994; Painter, 1996; Molz, Liu, and Szulga, 1997; Liu and Molz, 1997). The fLm model utilizes the Levy-stable family of probability distributions that have a canonical role in mathematical statistics similar to that of the Gaussian distribution, but have power law tails. These slowly decaying tails lead to diverging second and higher moments, but also make these distributions useful for modeling highly heterogeneous systems (Painter, 1996). However, for practical applications, truncation of Levy-stable distributions is generally needed such that simulated property values are bounded by the maximum and minimum values of measurements. Although other improved models of probability distributions have been proposed recently, that have both slowly decaying tails and a defined variance (Painter, 2001; Nakao, 2000), the relatively simple truncated fLm remains an attractive model for practical applications.

To employ fLm to characterize and simulate subsurface heterogeneities, it is desirable to have an efficient generating algorithm that is easy to understand and use. An algorithm that was proposed some time ago for generating the related Gaussian stochastic fractal known as fractional Brownian motion ( $\mathrm{fBm}$ ) is called successive random additions (SRA) (Voss, 1988; Saupe, 1988). This algorithm is easy and fast, has been subject to several 
suggested improvements, but continuing problems have been noted with the resulting correlation structure, especially for the higher dimensional versions (Caccia and others, 1997; McGaughey and Aithen, 2000). Because Levy-stable distributions do not have well-defined variances, the classical SRA algorithm, and other algorithms, for generating Gaussian fractals cannot be simply borrowed for generating fLm distributions. As a result, studies of algorithms for generating fLm are very limited in the literature. Painter (1996) published an algorithm based on generalized sequential simulation to simulate truncated fLm. This method was approximate and was not based on a random field model (Painter, 1998). A more rigorous method with conditional simulation was presented by Painter (1998) that again used sequential simulation. The sequential simulation method is inefficient because it requires solving a set of equations simultaneously for determining property values at each location. A more detailed discussion of issues related to using sequential simulation algorithm for generating fLm was recently presented by Gunning (2002). Owing to its computational efficiency and what should be a transparent derivation, it is logical to attempt to generalize SRA for fLm generation, but only if existing problems with the algorithm can be corrected. Herein we describe and correct the logical flaw that has persisted in the SRA algorithm, and then generalize the algorithm for simulating truncated fLm accurately in one or two dimensions.

\section{CORRECTION OF THE CLASSICAL SRA ALGORITHM FOR FBM}

We will state briefly the corrected SRA algorithm and then move on to the generalization to fLm. By doing this first for the Gaussian fractal, $\mathrm{fBm}$, analogoies with fLm will be more clear. One-dimensional fBm is defined as a random and single-valued 
function, $G(X)$, of the independent spatial or temporal variable, $X$, having stationary increments, $G(X+h)-G(X)$, over the distance (lag), $h$, with the increments displaying a Gaussian distribution with mean zero and variance, $\sigma^{2}$, for any h (Molz, Liu, and Szulga, 1997). Well known properties of the Gaussian distribution are that the summation of $\mathrm{N}$ independent random variables having a zero mean and variances, $\sigma_{\mathrm{i}}^{2}$, are still Gaussian with a standard deviation given by:

$\sigma=\left(\sum_{1}^{N} \sigma_{i}^{2}\right)^{1 / 2} \quad$ or in terms of variance, $\quad \sigma^{2}=\sum_{1}^{N} \sigma_{i}^{2}$

This property, along with the fact that a constant, A, multiplied by a Gaussian random variable with zero mean and variance, $\sigma_{1}^{2}$, is still Gaussian, but with $\sigma^{2}=A^{2} \sigma_{1}^{2}$, are the basic properties used in developing the traditional SRA algorithm given below.

The statistical self-affinity (scaling) of fBm may be expressed by relating the standard deviations of the increments, $G(X+h)-G(X)$, to the lag, h (Molz, Liu, and Szulga, 1997):

$\sigma^{2}(h)=\sigma^{2}(1) h^{2 H}$

where the scaling parameter, $\mathrm{H}$ (Hurst coefficient), is a constant.

To generate 1-D fBm having the above scaling, one begins with 2 points separated by a distance, $\mathrm{L}$, having initial values of zero. Assume that one wants to generate an $\mathrm{fBm}$ with $\mathrm{NN}=2^{n}+1$ data points. Then the following steps are required, as illustrated in Figure 1 ( Voss, 1988; Saupe, 1988): 
1. First add random numbers from $\mathrm{N}\left(0, \sigma_{0}{ }^{2} / 2\right)$ to the 2 end points, denoted by number 1 , where $\mathrm{N}\left(0, \sigma_{0}{ }^{2} / 2\right)$ stands for a Gaussian random number generator with a mean of 0 and a variance $\sigma_{0}^{2} / 2$.

2. Linearly interpolate the midpoint (denoted by number 2) value based upon the two end point values resulting from step 1 .

3. Add random numbers from $\mathrm{N}\left(0, \sigma_{1}^{2} / 2\right)$ with $\sigma_{1}^{2}=\frac{\sigma_{0}^{2}}{(2)^{2 H}}\left(1-\frac{2^{2 H}}{4}\right)$ to the midpoint and all other points;

4. Then, linearly interpolate between the 3 points obtained in step (3), denoted by number 3

5. Add random numbers from $\mathrm{N}\left(0, \sigma_{2}^{2} / 2\right)$ with $\sigma_{2}^{2}=\frac{\sigma_{1}^{2}}{2^{2 H}}=\frac{\sigma_{0}^{2}}{\left(2^{2 H}\right)^{2}}\left(1-\frac{2^{2 H}}{4}\right)$ to the two midpoints interpolated in step (4) and all other points.

6. Repeating the same process, keep performing linear interpolation and adding random numbers from $\mathrm{N}\left(0, \sigma_{n}^{2} / 2\right)$ with $\sigma_{n}^{2}=\frac{\sigma_{n-1}^{2}}{2^{2 H}}=\frac{\sigma_{0}^{2}}{\left(2^{2 H}\right)^{n}}\left(1-\frac{2^{2 H}}{4}\right)$ up to the nth level, which results in a total of $2^{n}+1$ points.

7. Although no new points are added, keep adding random numbers to each existing point from $\mathrm{N}\left(0, \sigma_{\mathrm{j}}^{2} / 2\right)$ with $\sigma_{j}^{2}=\frac{\sigma_{j-1}^{2}}{2^{2 H}}=\frac{\sigma_{0}^{2}}{\left(2^{2 H}\right)^{j}}\left(1-\frac{2^{2 H}}{4}\right) \quad$ for $\quad j=n+1, n+2, \ldots .$. $\mathrm{NM}$, where NM is a sufficiently large number such that $\sigma_{N M} / \sigma_{0}$ is negligible. Note that existing points refer to points added from Steps 1 to 6 . 
This final step is what has been missing from previous algorithms, and is required to obtain the proper variance structure at the nth, and nominally final, step of the algorithm. The reason for this, as well as a more detailed explanation for the entire algorithm, will be presented in the more general context of the Levy-stable distribution in the next section.

\section{FRACTIONAL LEVY MOTION (FLM)}

Again for convenience, we use initially one-dimensional (1D) fLm to demonstrate important fLm properties. Analogous to the Gaussian case, this stochastic function is defined as a random and single-valued function, $\mathrm{V}(\mathrm{X})$, of the independent spatial or temporal variable, $\mathrm{X}$, having stationary increments, $\mathrm{V}(\mathrm{X}+\mathrm{h})-\mathrm{V}(\mathrm{X})$, over the distance (lag) $\mathrm{h}$, with the increments displaying a (symmetric) Levy stable distribution of zero median for any h. A (symmetric) Levy-stable distribution is a generalization of a Gaussian distribution, and may be defined conveniently as the inverse Fourier transform of its characteristic function, since this inverse transform in general does not have an analytical expression (Samorodnitsky and Taqqu, 1994):

$f(x)=\frac{1}{\pi} \int_{0}^{\infty} \exp \left(-|C k|^{\alpha}\right) \cos (k x) d k$

where $\mathrm{C}$ is the width parameter, corresponding to the standard deviation of Gaussian distributions, and $\alpha$ is the Levy index. Such a distribution does not have a defined variance for $\alpha<2$, nor a defined mean for $\alpha<1$. (For $1 \leq \alpha \leq 2$ the zero mean and 
median are identical.) When $\alpha=2$, Equation (3) reduces to the well-known Gaussian distribution, so the Levy distribution contains the Gaussian distribution as a special case. Shown in Figure 2 are three Levy-stable distributions with different $\alpha$ values.

Similar to random variables following Gaussian distributions, the probability density distribution for the summation of $\mathrm{N}$ independent random variables, characterized by Levy-stable distributions with index $\alpha$, is still a Levy-stable distribution with the same index $\alpha$, and the following width parameter (Samorodnitsky and Taqqu, 1994)

$$
C=\left(\sum_{i=1}^{N} C_{i}^{\alpha}\right)^{1 / \alpha} \Rightarrow C^{\alpha}=\sum_{i=1}^{N} C_{i}^{\alpha}
$$

where $\mathrm{C}_{\mathrm{i}}$ is the width parameter for the ith independent random variable. However, it is the second relationship in terms of $\mathrm{C}^{\alpha}$ that forms the direct analogy with the variance for the Gaussian case and becomes the Gaussian variance rule when $\alpha=2$. As will be shown below, this is a fundamental property used for developing the SRA algorithm for fLm. Another fundamental property is that the random variable, $\mathrm{AZ}$, where $\mathrm{A}$ is a constant and $\mathrm{Z}$ is a random variable having Levy index $\alpha$ and width parameter $\mathrm{C}$, will also have a Levy index $\alpha$ but width parameter $\mathrm{AC}$, which when raised to the $\alpha$ power becomes $\mathrm{A}^{\alpha} \mathrm{C}^{\alpha}$ (Samorodnitsky and Taqqu, 1994).

The statistical self-affinity (scaling) of fLm may be expressed by relating the width parameters of the increments, $\mathrm{V}(\mathrm{X}+\mathrm{h})-\mathrm{V}(\mathrm{X})$, for different lags (h) (Painter, 1996):

$$
C(h)=C(1) h^{H} \quad \Rightarrow \quad C^{\alpha}(h)=C^{\alpha}(1) h^{\alpha H}
$$


where the scaling parameter $\mathrm{H}$ is a constant. (Again, the second relationship is the most direct analogy with the Gaussian case.) For $H=1 / \alpha$, the increments are independent (summing to a so-called Levy flight). For $1 / \alpha<\mathrm{H}<1$ and $0<\mathrm{H}<1 / \alpha(1<\alpha \leq 2), \mathrm{V}(\mathrm{X})$ has long-range positive and negative dependencies in the increments, respectively (Painter, 1996). Multidimensional fLm can also be easily defined by replacing the independent variable $\mathrm{X}$, used in the above discussion, with relevant location vectors.

\section{DEVELOPMENT OF AN SRA ALGORITHM FOR FLM}

The existing SRA algorithm was originally developed for Gaussian fractals. To our knowledge, a rigorously developed SRA algorithm for generating fLm, which automatically includes the Gaussian case, is not available in the literature. In this section, we first present the developed SRA algorithm for generating 1-D fLm and then discuss the logic of the algorithm. Extension of the algorithm to multidimensional fLm then follows.

To generate a 1-D fLm, one begins with two points separated by a distance, L, having initial values of zero (Fig. 1). Assume that one wants to generate a fLm with $N N=2^{\mathrm{n}}+1$ data points. Then the following steps are required: 
1. First add random numbers from $\mathrm{L}\left(0, \alpha, \sigma_{0}{ }^{\alpha} / 2\right)$ to the two end points, denoted by number 1 in Figure 2, where $\mathrm{L}\left(0, \alpha, \sigma_{0}{ }^{\alpha} / 2\right)$ stands for a Levy random number generator with a mean of zero, Levy index $\alpha$ and width parameter $\sigma_{0} / 2^{1 / \alpha}$.

2. Linearly interpolate the midpoint (denoted by number 2) value based on the two end point values resulting from step one.

3. Add random numbers from $\mathrm{L}\left(0, \alpha, \Delta_{1}^{\alpha} / 2\right)$ with $\Delta_{1}^{\alpha}=\frac{\sigma_{0}^{\alpha}}{2^{\alpha H}}\left(1-\frac{2^{\alpha H}}{2^{\alpha}}\right)$ to the middle point and all other points.

4. Linear interpolate between the " 3 " points obtained in the above step, denoted by number 3 .

5. Add random numbers from $L\left(0, \alpha, \Delta_{2}^{\alpha} / 2\right)$ with $\Delta_{2}^{\alpha}=\frac{\Delta_{1}^{\alpha}}{2^{\alpha H}}=\frac{\sigma_{0}^{\alpha}}{\left(2^{\alpha H}\right)^{2}}\left(1-\frac{2^{\alpha H}}{2^{\alpha}}\right)$ to the two midpoints interpolated from the above step and all other points.

6. Repeating the same process, keep performing linear interpolation and adding random numbers from $\mathrm{L}\left(0, \alpha, \Delta_{\mathrm{n}}^{\alpha} / 2\right)$ with $\Delta_{n}^{\alpha}=\frac{\Delta_{n-1}^{\alpha}}{2^{\alpha H}}=\frac{\sigma_{0}^{\alpha}}{\left(2^{\alpha H}\right)^{n}}\left(1-\frac{2^{\alpha H}}{2^{\alpha}}\right)$ up to the nth level, resulting in a total of $2^{\mathrm{n}}+1$ points.

7. Although no new points are added, continue adding random numbers from $\mathrm{L}(0, \alpha$, $\left.\Delta_{\mathrm{j}}^{\alpha} / 2\right)$ with $\Delta_{j}^{\alpha}=\frac{\Delta_{j-1}^{\alpha}}{2^{\alpha H}}=\frac{\sigma_{0}^{\alpha}}{\left(2^{\alpha H}\right)^{j}}\left(1-\frac{2^{\alpha H}}{2^{\alpha}}\right)$ to each existing point for $\mathrm{j}=\mathrm{n}+1, \mathrm{n}+2, \ldots$, $\mathrm{NM}$, where $\mathrm{NM}$ is a sufficiently large number such that $\mathrm{C}_{\mathrm{NM}} / \mathrm{C}_{0}$ is negligible. 
Note that when $\alpha=2$, steps 1-7 are identical to those in the SRA algorithm for Gaussian fractals described previously. Also note that we added the new step (step 7) in the current algorithm, which is essential for generating properly correlated and scaling fLm (as well as fBm), as will be discussed later. To understand why the developed SRA algorithm can generate selected fLm distributions and to understand the error term in the construction procedure, one needs to know how the relevant formulations used in the algorithm were derived.

After step 1, based on Equation (4), the increments (differences of point values) of $\mathrm{V}(\mathrm{X})$ are characterized by a Levy-stable distribution with width parameter $\mathrm{C}\left(\mathrm{L} / 2^{0}\right)$ derived from

$C^{\alpha}\left(L / 2^{0}\right)=\sigma_{0}^{\alpha}$

where $\mathrm{L} / 2^{0}$ is the lag for the increments of $\mathrm{V}(\mathrm{X})$ after step 1 . After steps 2 and 3 , the $\alpha$ power width parameter for the $\mathrm{V}(\mathrm{X})$ increments with $\operatorname{lag} \mathrm{L} / 2^{0}$ becomes

$$
C^{\alpha}\left(L / 2^{0}\right)=\sigma_{0}^{\alpha}+\Delta_{1}^{\alpha}
$$

where $\Delta_{1}{ }^{\alpha}$ results from adding random numbers from $L\left(0, \alpha, \Delta_{1}^{\alpha} / 2\right)$. At the same time, the width parameter for the $\mathrm{V}(\mathrm{X})$ increments with lag $\mathrm{L} / 2^{1}$ becomes

$$
C^{\alpha}\left(L / 2^{1}\right)=\sigma_{1}^{\alpha}=\left(\frac{\sigma_{0}}{2}\right)^{\alpha}+\Delta_{1}^{\alpha}
$$


where the term $\left(\sigma_{0} / 2\right)^{\alpha}$ results from the linear interpolation (step 2) and the term $\Delta_{1}^{\alpha}$ again results from the addition of random numbers. For reasons to become clear later on (see Equation (14)), we need

$$
\sigma_{1}^{\alpha}=\frac{1}{2^{\alpha H}} \sigma_{0}^{\alpha}
$$

Combination of Equations (8) and (9) results in

$$
\Delta_{1}^{\alpha}=\frac{\sigma_{0}^{\alpha}}{2^{\alpha H}}\left(1-\frac{2^{\alpha H}}{2^{\alpha}}\right),
$$

which is the equation used in step 3 of the fLm algorithm. As the algorithm proceeds, and we keep adding random numbers to all points, the $\mathrm{C}^{\alpha}$ values at all levels $(\mathrm{L}, \mathrm{L} / 2, \mathrm{~L} / 4, \ldots$, etc.) keep changing, and one must keep track of this in order for the final result to have the proper scaling. Following the same logic used to derive Equations (7) through (9), after step 7, one obtains

$C^{\alpha}\left(L / 2^{i}\right)=\sigma_{i}^{\alpha}+\sum_{j=i+1}^{N M} \Delta_{j}{ }^{\alpha}$

$\sigma_{i}^{\alpha}=\left(\frac{1}{2^{\alpha H}}\right)^{i} \sigma_{0}^{\alpha}$ 
$\Delta_{i+1}^{\alpha}=\frac{\sigma_{0}^{\alpha}}{\left(2^{\alpha H}\right)^{i+1}}\left(1-\frac{2^{\alpha H}}{2^{\alpha}}\right)$

where $\mathrm{i}=0,1, \ldots \ldots, \mathrm{n}$. Combining Equations $(11)-(13)$, one may derive the $\alpha$-power width parameter ratios:

$$
\frac{C^{\alpha}\left(L / 2^{i+1}\right)}{C^{\alpha}\left(L / 2^{i}\right)}=\frac{1}{2^{\alpha H}}+\frac{\Delta_{N M}^{\alpha}}{\sigma_{i}^{\alpha}+\sum_{j=i+1}^{N M} \Delta_{j}^{\alpha}}
$$

This general result, and its Gaussian counterpart, is what motivates Step 7 in the modified SRA algorithm. Only if the second term on the right hand side of Equation (14) can be ignored, will the SRA algorithm satisfy the desired scale relationship implied by Equation (5). However, in the traditional SRA algorithm without step $7(\mathrm{NM}=\mathrm{n})$, the second term may be sufficiently large such that the scaling relationship is not obtained. This is the reason why the traditional SRA algorithm has been found to provide stochastic fractal distributions of questionable scaling and correlation properties (Caccia and others, 1997; McGaughey and Aitken, 2000). Our new step 7 in the current version of SRA can essentially eliminate the problem, which will be demonstrated later. From Equation (14), one may easily understand why Equations (9) and (12) are needed to produce the correct scale relationships. All random additions of the algorithm must be considered in superposition in order to motivate the correct steps.

McGaughey and Aitken (2000) indicated that traditional SRA resulted in nonstationary increments for 1-D fBm. They investigated correlation functions of time series generated by the SRA for two cases. These two cases correspond to correlation for a 
single-sample time lag for the difference between two points and their interpolated midpoint and correlation for a single time lag involving two interpolated midpoints and one point between the two midpoints, respectively. Difference between two correlationfunction values is given (McGaughey and Aitken, 2000)

$\varepsilon=\sum_{k=1}^{N M-l} \frac{3}{4^{k}} \frac{\sigma^{2} N M-k}{4}$

where 1 is the iteration the point between the two midpoints was initially added for the second case. The difference between correlation values for the same lag is an indication of non-stationary increments. Detailed derivation of Equation (15) can be found in McGaughey and Aitken (2000). (Note that different notions are used here.) From Step 6 in the corrected SRA procedure for generating $\mathrm{fBm}$ (Section 2), the variance in Equation (15) can be determined by

$$
\sigma^{2} N M-k=\frac{\sigma^{2} 0}{2^{2 H(N M-k)}}\left(1-\frac{2^{2 H}}{4}\right)
$$

Combining Equations (15) and (16) yields:

$\varepsilon=\frac{\sigma_{0}^{2}}{2^{(N M) H}}\left(1-\frac{2^{2 H}}{4}\right) \sum_{k=1}^{N M-l} \frac{1}{2^{2 k(1-H)}}$

In the traditional SRA algorithm without step $7(\mathrm{NM}=\mathrm{n})$, the $\varepsilon$ may be sufficiently large such that the increments are non-stationary. However, with the new step 7, we can use large NM to make $\varepsilon$ practically negligible. In other words, our new step 7 in the current version of SRA can essentially eliminate the problem regarding non-stationary increments at least for 1-D fBm. However, it is mathematically difficult to prove that the corrected SRA can also eliminate the problem for 1-D fLm (because the correlation 
function cannot be defined for fLm), although we intuitively expect it to be the case based on similarities between $\mathrm{fBm}$ and fLm. Further research is needed to completely resolve this issue. On the other hand, in many practical applications (e.g., simulation of heterogeneous permeability distributions), one is more concerned with if the scaling relation (Equation (5)) is correctly simulated (Molz, Liu, and Szulga, 1997; Painter, 1996). Slightly non-stationary increments are not expected to significantly affect simulated heterogeneous distributions.

The SRA algorithm for 1-D fLm presented above may be extended to multidimensional cases. As an example, we briefly demonstrate how the SRA algorithm can be used for generating isotropic two-dimensional (2-D) fLm distributions. We begin with four points (a, b, c, and d in Figure 3). They are separated by a distance L in the vertical and horizontal directions, respectively. They have initial values of zero. Similar to step 1 for generating 1-D fLm, we add random numbers from $\mathrm{L}\left(0, \alpha, \sigma_{0}{ }^{\alpha} / 2\right)$ to the four points. As in step 2 for generating 1-D fLm, we linearly interpolate the midpoints (such as point D in Figure 3) in both vertical and horizontal directions. Then we calculate a value for the center point $\mathrm{E}$ by averaging the values of the four corner points. Similar to step 3 for 1-D fLm, we add random numbers from $\mathrm{L}\left(0, \alpha, \Delta_{1}{ }^{\alpha} / 2\right)$ with $\Delta_{1}{ }^{\alpha}=(1-$ $\left.2^{\alpha \mathrm{H}} / 2^{\alpha}\right) \sigma_{0}{ }^{\alpha} / 2^{\alpha \mathrm{H}}$ to the middle points, the center point and all other points. The following steps are similar to those used for generating 1-D fLm, except that for each step there are center points to be added in addition to middle points.

It may not be straightforward to understand why random numbers with the same statistical parameters are added to both center points and middle points when these points are added. To understand this issue, let us assume that random numbers with different 
statistics need to be added to the center point $\mathrm{E}$ and the middle points in step 3 (see Fig. 3). After that step, for the $V$ increment between point a and the middle point $D$, we have

$$
C^{\alpha}\left(L / 2^{1}\right)=\sigma_{1}^{\alpha}=\left(\frac{\sigma_{0}}{2}\right)^{\alpha}+\Delta_{1}^{\alpha}=\left(\frac{1}{2^{\alpha H}}\right) \sigma_{0}^{\alpha}
$$

Obviously, the same relation holds for all other middle points and their adjacent points in the vertical or horizontal directions. Let us assume further that we add a random number from $\mathrm{L}\left(0, \alpha, \Delta_{\mathrm{IE}}^{\alpha} / 2\right)$ to the center point. As a result, the width parameter $\left(\mathrm{C}_{\mathrm{E}}\right)$ characterizing the increments of the random function, $\mathrm{V}$, between the middle point $\mathrm{D}$ and the center point $\mathrm{E}$ becomes

$$
C_{E}^{\alpha}\left(L / 2^{1}\right)=\left(\frac{\sigma_{0 E}}{2}\right)^{\alpha}+\frac{\Delta_{1}^{\alpha}}{2}+\frac{\Delta_{1 E}{ }^{\alpha}}{2}
$$

where $\sigma_{0 \mathrm{E}}$ is the width parameter characterizing the $\mathrm{V}$ increments between the middle points and the center point before the random numbers were added to the two points. Specifically, the $\mathrm{V}$ increment between points $\mathrm{E}$ and $\mathrm{D}$ is equal to $0.5\left[\left(\mathrm{~V}_{\mathrm{d}}-\mathrm{V}_{\mathrm{a}}\right)+\left(\mathrm{V}_{\mathrm{c}}-\mathrm{V}_{\mathrm{b}}\right)\right]$, where $V_{i}(i=a, b, c$ and $d)$ is a random function value at point $i$ for the given step (Figure 3). If we assume that $\left(\mathrm{V}_{\mathrm{d}^{-}}-\mathrm{V}_{\mathrm{a}}\right)$ and $\left(\mathrm{V}_{\mathrm{c}^{-}}-\mathrm{V}_{\mathrm{b}}\right)$ are independent, $\sigma_{0 \mathrm{E}}$ will be the same as $\sigma_{0}$. This is the only additional assumption needed to extend the SRA algorithm from 1-D to 2-D cases. As will be indicated in our numerical tests, use of this assumption gives satisfactory simulation results. Considering the isotropy of the random field and to 
satisfy the scale relationship for $\mathrm{fLm}$, we need $\mathrm{C}\left(\mathrm{L} / 2^{1}\right)$ given in (18) to equal to $\mathrm{C}_{\mathrm{E}}\left(\mathrm{L} / 2^{1}\right)$ given in (19), which results in

$\Delta_{1}=\Delta_{1 E}$

Similar relations can also be obtained for other steps. It proves that random numbers with the same statistical parameters should be added to both middle points and the center points at relevant steps of the SRA algorithm.

A similar procedure may be used to extend SRA from 1-D to 3-D cases. Note that we only discuss the generation of isotropic fLm distributions in this paper. Methods for transforming isotropic fLm distributions to anisotropic ones can be found in $\mathrm{Lu}, \mathrm{Molz}$, and Liu (2003).

\section{NUMERICAL VALIDATION OF THE MODIFIED/GENERALIZED SRA}

\section{ALGORITHM}

To examine the validity of the developed SRA algorithm, we generated 20 1-D fLm distributions of length 257 by choosing $H=0.160, \alpha=1.25, \mathrm{NM}=62$ and $\sigma_{0}=0.15$. (Shown in Figure 4 is one of these fLm distributions.) Note that the length corresponds to $2^{\mathrm{n}}+1(\mathrm{n}=8$ here $)$. Then we analyzed fLm parameters for the 20 generated distributions using the method of Fama and Roll (1972). Shown in Figure 5 is the width parameter, C, as a function of lag. The fitted $\mathrm{H}$ value of 0.159 is very close to the input value, indicating that the correct scale relation was simulated. We also analyzed 20 1-D distributions generated with the same input parameters but without using the new step 
(step 7 in Section 4) that we added to the SRA algorithm. The resultant $H$ value is 0.333 (Fig. 6) that is considerably larger than the input value of 0.160 . This confirms previous findings that the traditional SRA algorithm may generate fractal distributions nonrepresentative of the input parameters, often necessitating a trial and error procedure in order to get a pre-selected H value (Caccia and others, 1997; McGaughey and Aitken, 2000). The fact that the resulting $H$ values for distributions generated without using the new step are larger than input values may be easily explained based on Equation (14) (when the second term on the right hand side of the equation is not negligible). As indicated in Figure 5, our improved SRA algorithm does not have this problem. It is critical to correctly simulate the scale relations for modeling subsurface heterogeneity. Numerical results (not shown here) also indicate that the developed SRA algorithm is able to correctly generate $\mathrm{fBm}$ distributions (including those for $\mathrm{H}>0.5$ ). This is expected because $\mathrm{fBm}$ is a special case of $\mathrm{fLm}$.

Shown in Figure 7 is a simulated 2-D fLm distribution with a side length of 257 . The input parameters are $\mathrm{H}=0.200, \alpha=1.25, \mathrm{NM}=62$ and $\sigma_{0}=0.15$. Analysis of the distribution in the horizontal direction results in $\mathrm{H}=0.203$, that again is very close to the input $\mathrm{H}$ value (Fig. 8), indicating that our SRA algorithm can also generate satisfactory multidimensional fLm distributions.

When it is necessary to obtain a fLm distribution with a given $\mathrm{C}_{0}$ (Equation (5)) rather than $\sigma_{0}$, there are two options. One may be to determine $\sigma_{0}$ directly from the given $\mathrm{C}_{0}$ from Equations (5) and (11) to (13). The other option is to adjust input $\sigma_{0}$ values through several trial runs until the correct $C_{0}$ value is obtained (Due to the efficiency of 
this algorithm, trial runs may be accomplished rapidly). A further discussion of this issue can be found in Lu, Molz, and Liu (2003).

Note that for the numerical test results presented in Figures 4-8, truncation of the simulated random function values was performed. If a simulated value at a location is larger (smaller) than 3.5 plus the mean of all simulated values ( -3.5 plus the mean), the value is changed to 3.5 plus the mean (-3.5 plus the mean). Different truncation schemes may be used for practical applications. Figure 9 shows the Levy index analyzed with the method of Fama and Roll (1971) for the 2-D fLm distribution. As a result of truncation, the index is not a constant, but scale (lag) dependent. This is consistent with previous simulation results of Painter (1998). More importantly, this behavior is consistent with the results of measured data analysis for $\log (\mathrm{K})$ (Liu and Molz, 1997).

\section{CONCLUSIONS}

A modified and generalized successive random additions (SRA) algorithm has been developed for simulating subsurface heterogeneity and other spatial or temporal distributions characterized by fLm or fBm. SRA, developed initially for generating Gaussian fractals associated with a well-defined variance, has been corrected and extended rigorously to generating fLm distributions in which variances are not defined. In doing this, use was made of the mathematical analogy that exists between the second order statistical moment of a Gaussian distribution and the highest order ( $\alpha$ order) statistical moment that is defined for a Levy-stable distribution. The generalized SRA algorithm is validated through numerical tests, wherein it is shown that a new step, derived from considering superposition of all random numbers added repeatedly to all 
points, overcomes the problem observed previously that the traditional SRA algorithm often produces Gaussian fractals having poor scaling and correlation properties. Note that the SRA can only be used to generate nonconditional spatial distributions. How to approximately develop conditional fLm distributions based on the corresponding nonconditional distributions and field observations was presented by Lu, Molz, and Liu (2003). Also note that the developed SRA algorithm can be used only for a structured grid system. An extension of the algorithm to a general unstructured grid needs further investigations. 


\section{ACKNOWLEDGMENT}

We are indebted to Dr. Stefan Finsterle at Lawrence Berkeley National Laboratory for his critical and careful review of a preliminary version of this manuscript. We also appreciate constructive comments from two anonymous reviewers. This work was supported by the Director, Office of Civilian Radioactive Waste Management, U.S. Department of Energy, through Memorandum Purchase Order EA9013MC5X between Bechtel SAIC Company, LLC and the Ernest Orlando Lawrence Berkeley National Laboratory (Berkeley Lab). The support is provided to Berkeley Lab through the U.S. Department of Energy Contract No. DE-AC03-76SF00098. 


\section{REFERENCES}

Caccia, D.C., Percival, D., Cannon, M. J., Raymond, G., Bassingthwaigthe, J. B., 1997, Analyzing exact fractal time series: Evaluating dispersion analysis and rescaled range methods: Physica A, v. 246, no. 3-4, p. 609-632.

Fama, E., and Roll, R., 1972, Parameter estimates for symmetric stable distributions: Jour. Am. Stat. Assoc., v. 66, no. 334, p. 331-338.

Gunning, J., 2002, On the use of multivariate Levy-stable random field models for geological heterogeneity: Math. Geol., v. 34, no. 1, p. 43-62.

Liu, H.-H., and Molz, F.J., 1997, Comment on "Evidence for non-Gaussian scaling behavior in heterogeneous sedimentary formations" by S. Painter: Water Resour. Res., v. 33, no. 4, p. 907-908.

Lu, S., Molz, F.J., and Liu, H.-H., 2003, An efficient, three-dimensional, anisotropic, fractional Brownian motion and truncated fractional Levy motion simulation algorithm based on successive random additions: Computers and Geosciences, v. 29, no.1, p. 15-25.

McGaughey, D. R., and Aithen, G. J. M., 2000, Statistical analysis of successive random additions for generating fractal Brownian motion: Physica A, v. 277, no, 1-2, p. 25-34. 
Molz, F.J., Liu, H .H., and Szulga, J., 1997, Fractional Brownian motion and fractional Gaussian noise in subsurface hydrology: A review, presentation of fundamental properties, and extension: Water Resour. Res., v. 33, no. 10, p. 2273-2286.

Nakao, H., 2000, Multi-scaling properties of truncated Levy flight: Phys. Lett. A, v. 266, no. 4-6, p. 282-289.

Painter, S., and Paterson, L., 1994, Fractional Levy motion as a model for spatial variability in sedimentary rock: Geophysical research letters, v. 21, no. 25, p. 2857-2860.

Painter, S., 1996, Stochastic interpolation of aquifer properties using fractional Levy motion: Water Resour. Res., v. 32, no. 5, p. 1323-1332.

Painter, S., 1998, Numerical method for conditional simulation of Levy random fields: Math. Geol., v. 30, no. 2, p. 163-179.

Painter, S., 2001, Flexible scaling model for use in random field simulation of hydraulic conductivity: Water Resour. Res., v. 37, no. 5, p. 1155-1164.

Samorodnitsky, G., and Taqqu, M. S., 1994, Stable non-Gaussian random processes: Chapman \& Hall, 632 p. 
Saupe, D., 1988, Algorithms for random fractals: In Peitgen, H.-O. and Saupe, D. (Eds), The Science of Fractal Images, Springer-Verlag, New York, p. 71-136.

Voss, R. F, 1988, Fractal in nature: From characterization to simulation: In Peitgen, H.O. and Saupe, D. (Eds), The Science of Fractal Images, Springer-Verlag, New York, p. 21-70. 


\section{Figures}

Figure 1. Schematic diagram showing the steps for the 1-D SRA algorithm.

Figure 2. Levy-stable distributions with the same $\mathrm{C}$ value $(\mathrm{C}=1)$ and different Levy index ( $\alpha$ ) values. Note that $\alpha=2$ corresponds to the Gaussian distribution.

Figure 3. Schematic diagram illustrating the 2-D SRA algorithm.

Figure 4. A 1-D fLm distribution.

Figure 5. Width parameter $\mathrm{C}$ values (as a function of lag) calculated from simulated1-D fLm distributions.

Figure 6. Width parameter $\mathrm{C}$ values (as a function of lag) calculated from simulated1-D fLm distributions generated using the SRA algorithm without the new step.

Figure 7. A 2-D fLm distribution generated with the generalized SRA algorithm

Figure 8. Width parameter $\mathrm{C}$ values (as a function of lag along the horizontal direction) calculated from the simulated 2-D fLm distribution.

Figure 9. Estimated Levy index values as a function of lag along the horizontal direction for the simulated 2-D fLm distribution. 


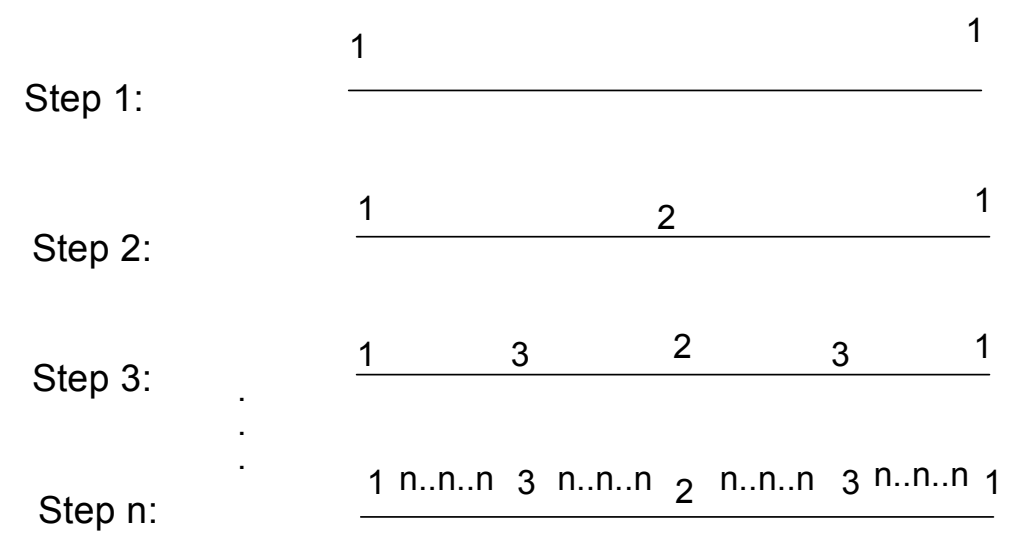




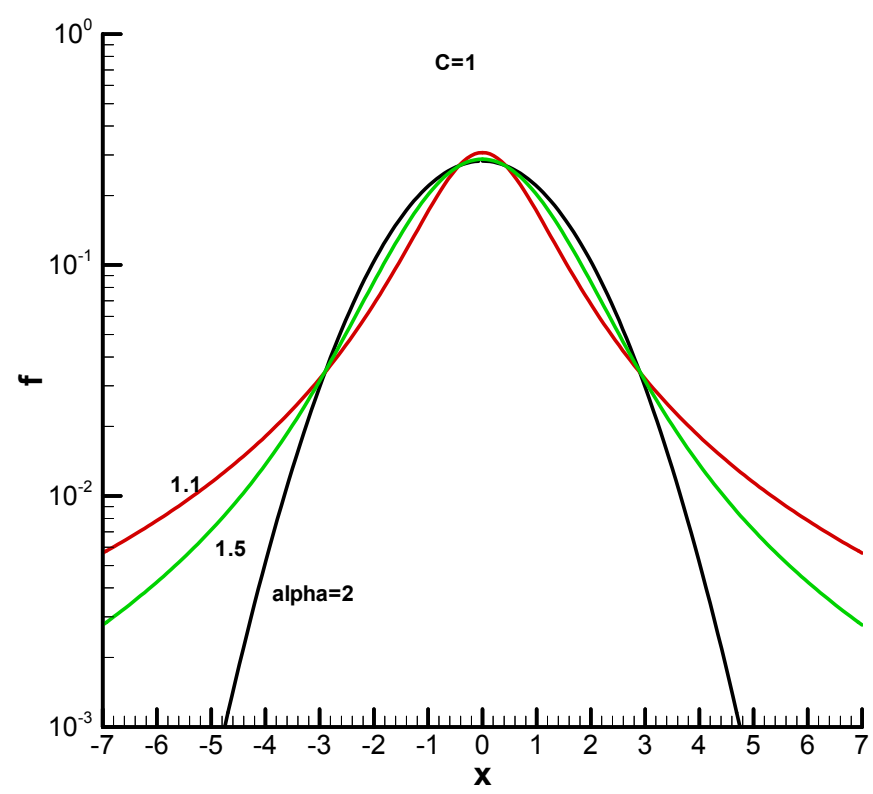




\begin{tabular}{|l|l|}
\hline a & $\mathbf{D}$ (middle point) b \\
& \\
\hline & $\mathbf{E}$ (center point) \\
& \\
\hline d & $\mathbf{c}$ \\
\hline
\end{tabular}




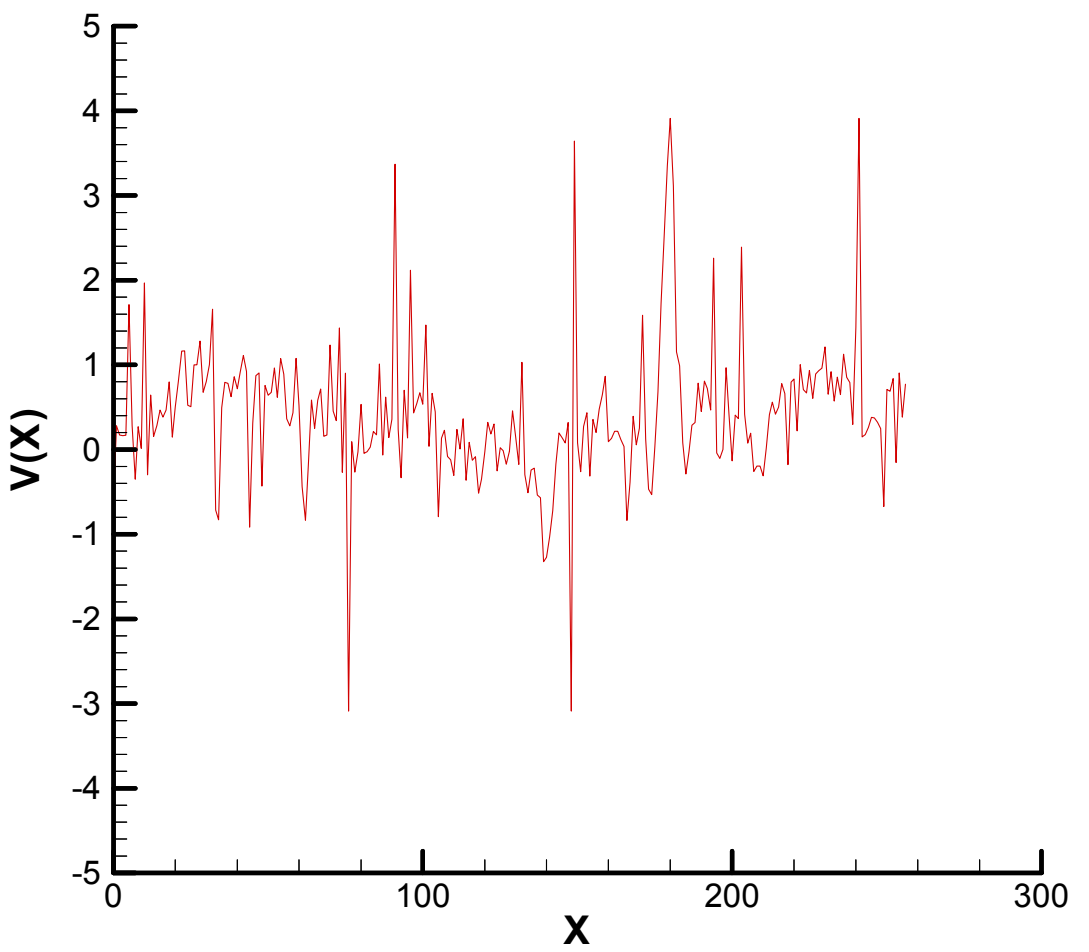




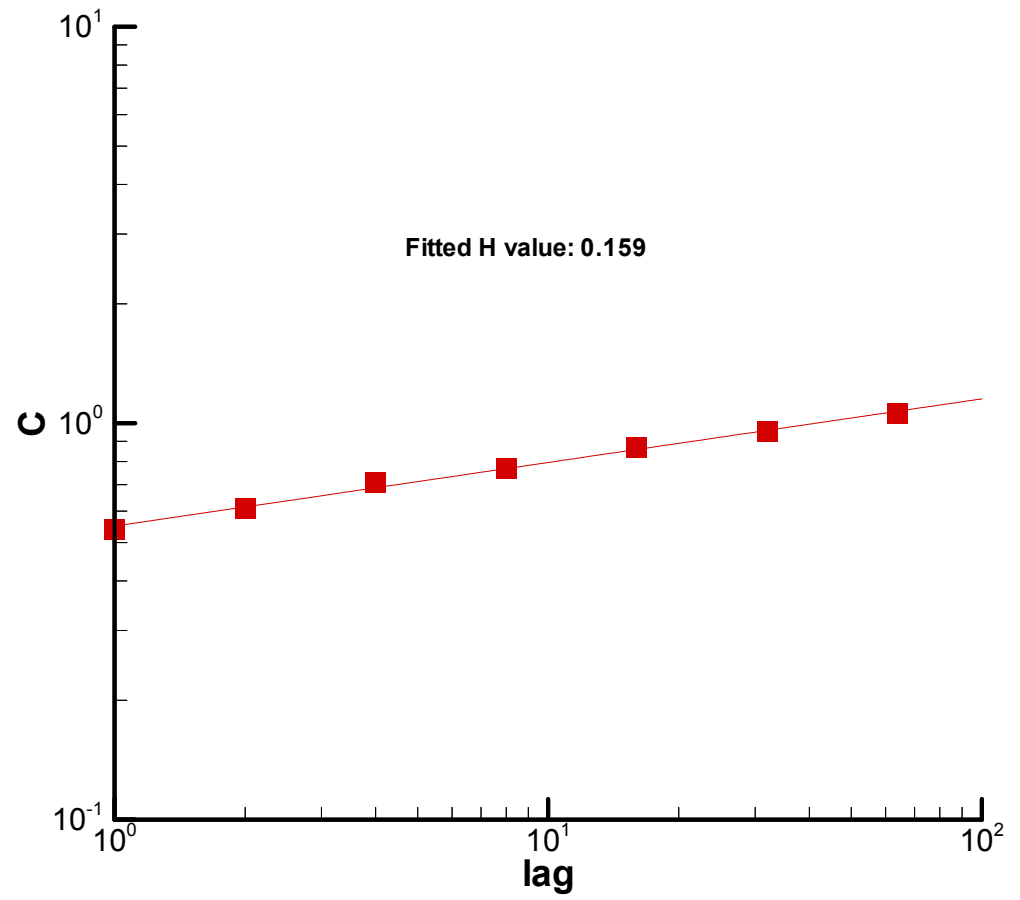




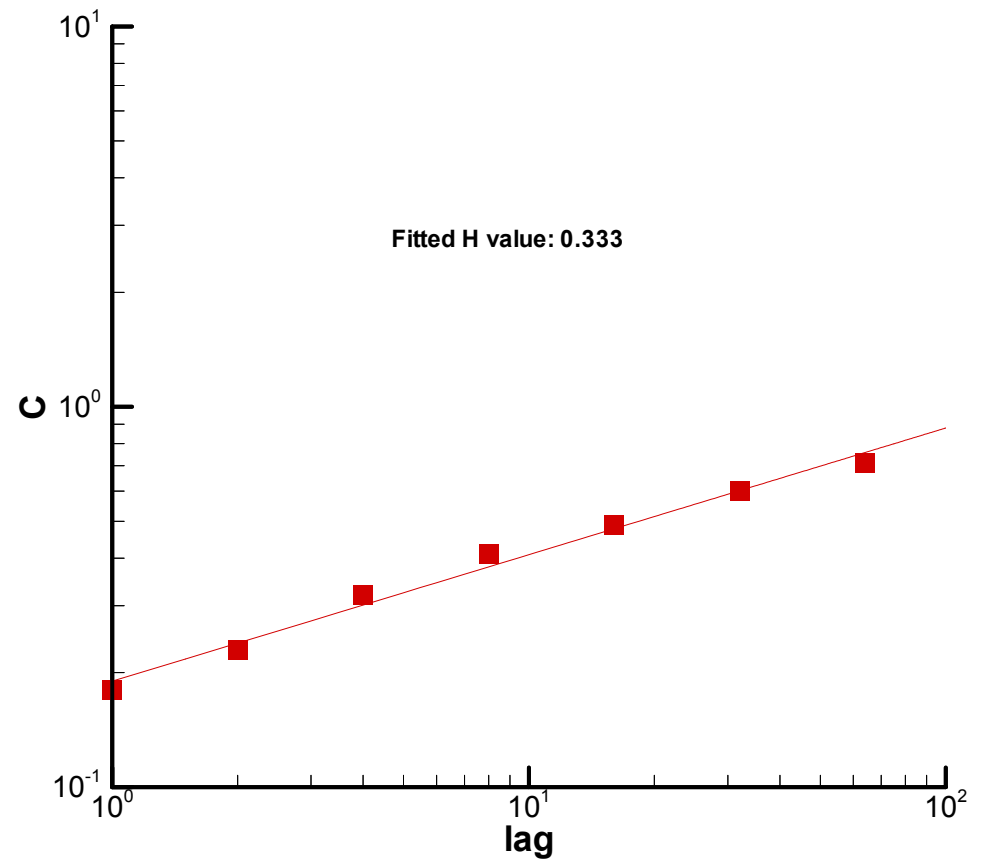




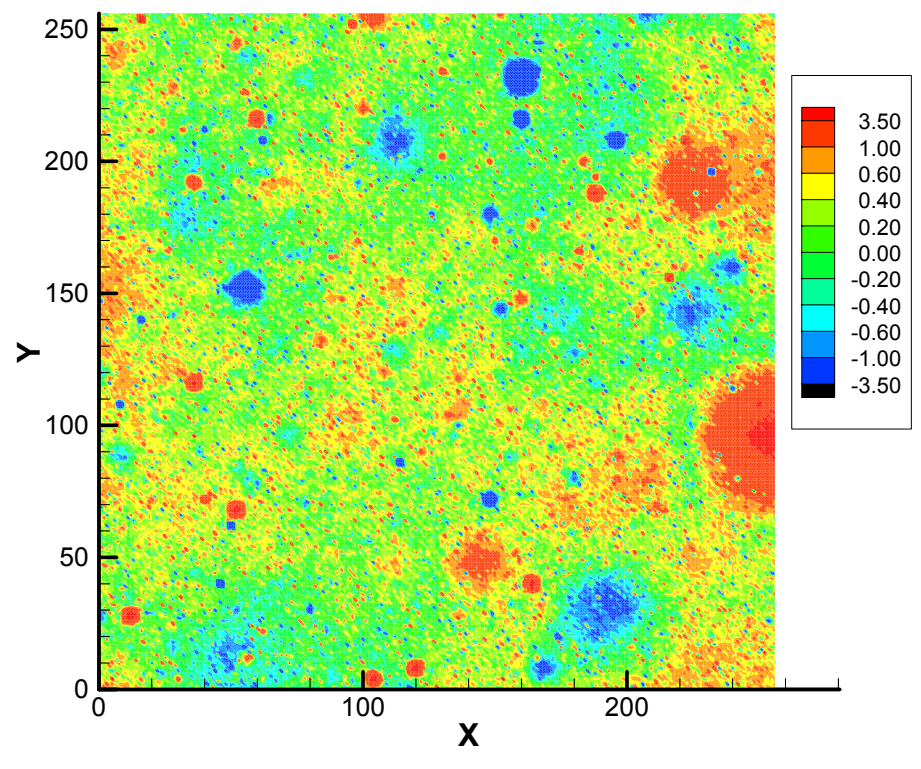




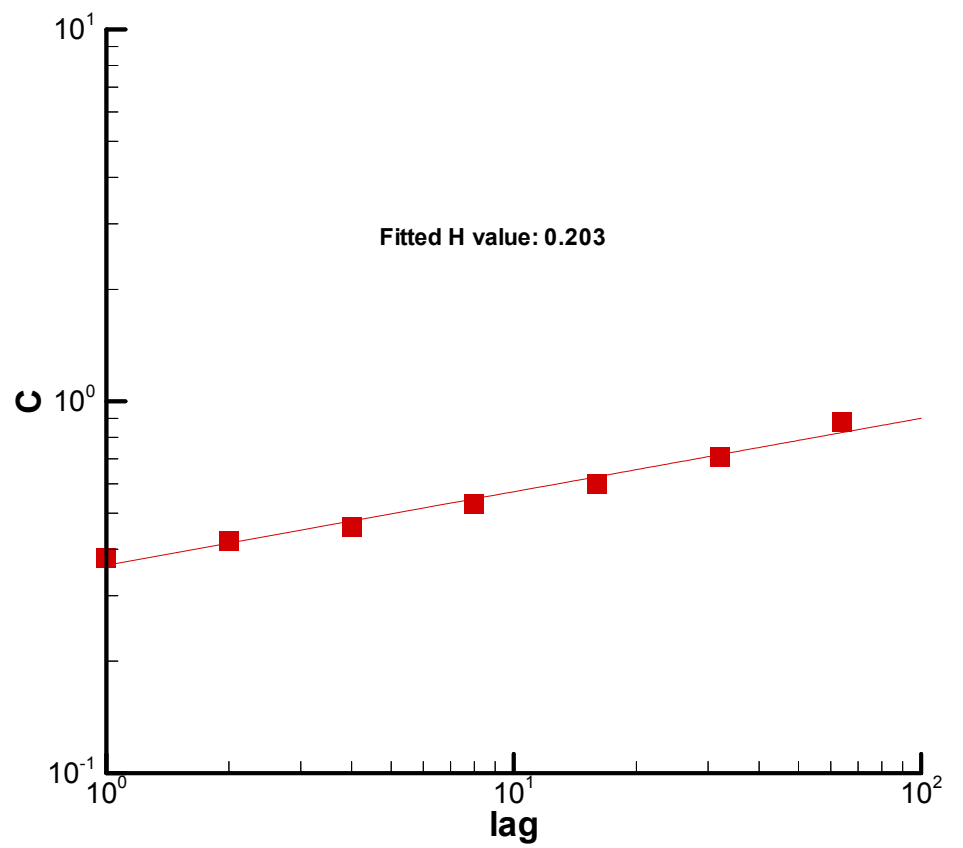




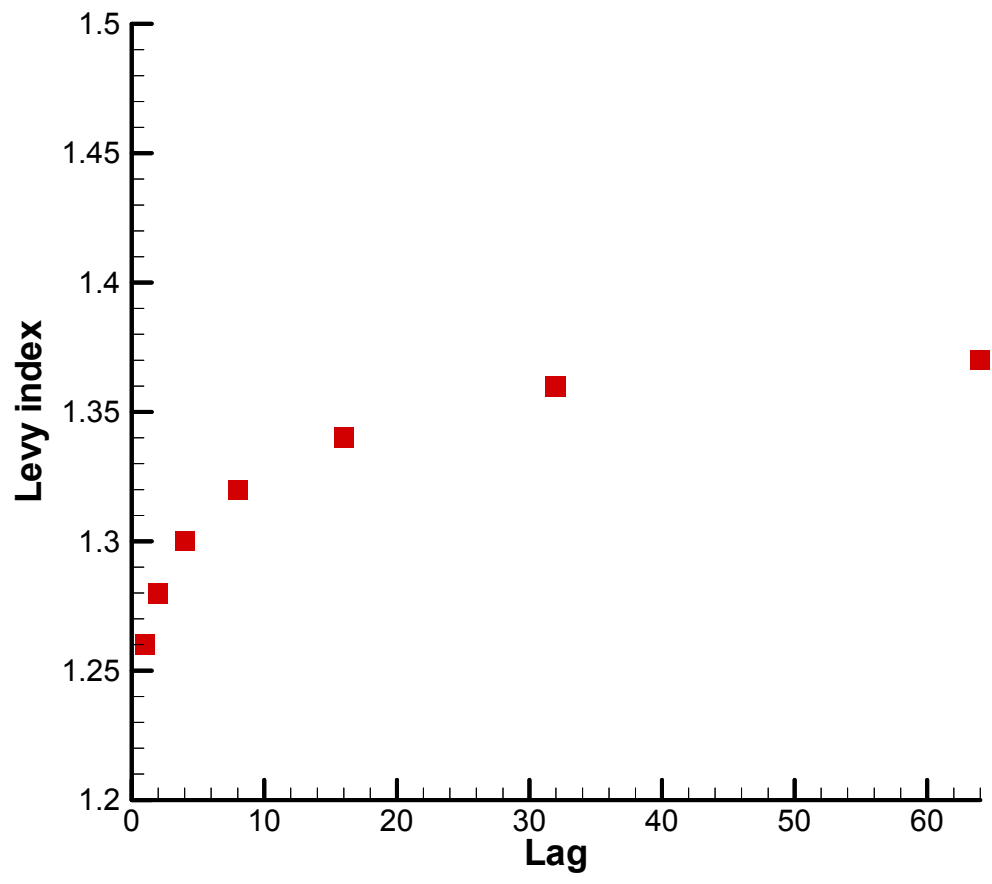

